\title{
Modular Control-Loop Detection
}

\author{
Petra Malik Robi Malik \\ Department of Computer Science, University of Waikato \\ Hamilton, New Zealand \\ \{petra,robi\}@es.waikato.ac.nz
}

\begin{abstract}
This paper presents an efficient algorithm to detect control-loops in large finite-state systems. The proposed algorithm exploits the modular structure present in many models of practical relevance, and often successfully avoids the explicit synchronous composition of subsystems and thereby the state explosion problem. Experimental results show that the method can be used to verify industrial applications of considerable complexity.
\end{abstract}

\section{INTRODUCTION}

Supervisory control theory is centred on the setting of a supervisor or controller interacting with a plant, which typically represents a technical system to be controlled. The theory provides fundamental results about the existence of supervisors, and algorithms that automatically synthesise supervisors satisfying certain properties [1], [2].

Ever more controllers are implemented in software, either running on dedicated programmable logic circuits (PLC) or on multi-purpose computers, so it is highly desirable to construct control software from models that have been obtained using supervisory control theory. However, traditional supervisory control is situated on a higher level of abstraction and does not provide all the details needed to actually implement a controller for physical devices. It has been recognised by several researchers [3]-[5] that, in order to implement a controller, its behaviour needs to satisfy additional properties that are not covered by traditional supervisory control theory.

One of these properties is the absence of control-loops [6], [7]. In many technical applications, a set of control actions can be identified. These correspond to commands generated by the controller and sent to the physical device or plant to achieve the desired behaviour. A system is control-loop free, if the controller never tries to generate an infinite sequence of control actions in response to any finite input from the plant. Clearly, this is a very desirable property of most controllers implemented in software.

This paper proposes an efficient algorithm to check whether a complex system is control-loop free. The proposed algorithm, which is based on results from [7], is an adaptation of a very efficient algorithm [8] to check safety properties of discrete event systems. The method exploits the modular structure of discrete event system models to identify and check subsystems, such that the results of checking the subsystems can give conclusions about the properties of the entire system. Counterexamples are used for guidance, to augment these subsystems if needed.
Existing model checking techniques for CTL [9] can also be used to check whether a system is control-loop free, and, using symbolic representations [10], have been used successfully to verify models of considerable complexity. Abstractions [11] can enhance the performance of these algorithms. This paper proposes an alternative approach that exploits the modular structure present in most discrete event models, which can be combined with and further enhance the existing methods.

In the following, section II presents a motivating example to clarify the need for control-loop detection. Section III gives the notations and definitions used throughout the rest of the paper. The problem of checking for control-loops is defined in section IV, where a basic algorithm is given. Then, section $\mathrm{V}$ presents the results underlying the modular control-loop detection algorithm that is the centre of this paper. Section VI describes the algorithm, and section VII lists experimental results. Finally, section VIII adds some concluding remarks.

\section{Dosing TANK ExAMPLE}

This section illustrates the idea of checking for controlloops using the example of a dosing unit in a chemical batch plant, used to supply a defined amount of liquid material to a subsequent process. It consists of a tank, an inlet valve, an outlet valve, and two sensors to check the filling level of the tank. The following is a simplified version of a system introduced in [12].

The two sensors, $\mathbf{S 1}$ at the bottom and $\mathbf{S 2}$ at the top of the tank, can either be on or off, indicating whether the tank has reached the corresponding filling level. Their state changes are modelled as events s1_on, s1_off, s2_on, and s2_off. The user can request the process of filling and emptying the tank to be started (req_start), or suspended (req_stop). To meet these requests, the controller has to open or close the inlet and the outlet valves appropriately, using control actions open_in, close_in, open_out, and close_out.

The possible behaviour of this plant is modelled by the automata sensors, requests, in, and out in fig. 1. A controller has been designed using additional automata to constrain the behaviour of the plant and satisfy certain requirements. Automaton no_flow ensures that liquid never flows through the tank, i.e., that the two valves are never open at the same time. Automaton req_spec guarantees that discharging or filling of the tank can only start when a request is present, i.e., after req_start has occurred. Finally, to provide 

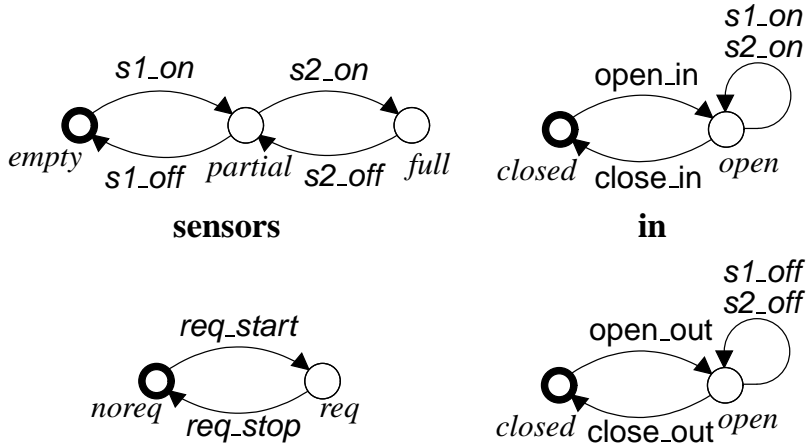

requests

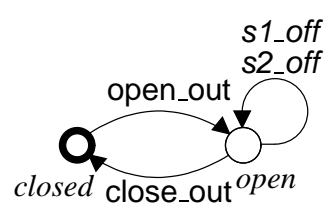

out

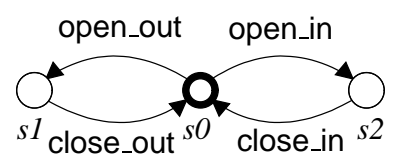

no_flow

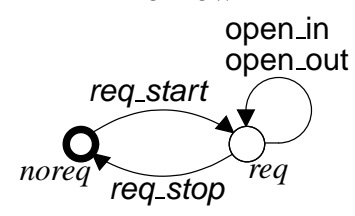

req_spec open_out

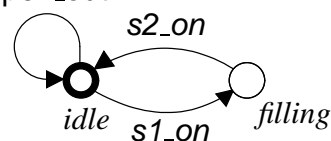

empty_spec

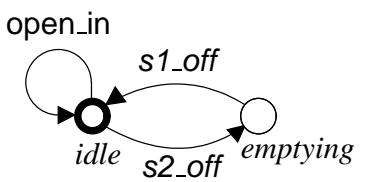

fi ll spec

Fig. 1. Automata for dosing tank example.

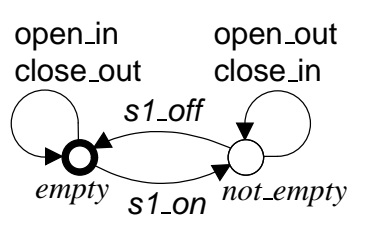

empty_spec'

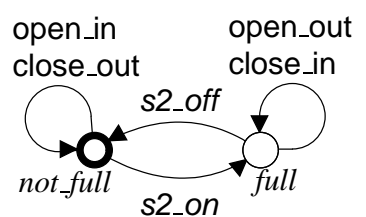

fi ll spec'

Fig. 2. Additional specifi cations for dosing tank example.

the correct amount of liquid to the subsequent process, the tank must always be completely filled, i.e., until sensor $\mathbf{S 2}$ goes on, before discharging can start (empty_spec), and completely emptied before filling can start (fi ll spec).

The question is whether a controller can be constructed that opens and closes the valves appropriately to yield the behaviour of the dosing unit as obtained when synchronising all the automata in fig. 1. A check for control-loops shows that this is not feasible. The automata can execute the trace

$$
\text { req_start open_in close_in open_in close_in ... }
$$

where the inlet valve is opened and closed indefinitely. While such behaviour does not violate the requirements given so far, it is very undesirable when executed by an actual controller.

To avoid this problem, additional specifications as shown in fig. 2 can be used to constrain the model further: the inlet valve can only be opened if the tank is empty and closed if the tank is full, and the outlet valve can only be opened if the tank is full and closed if the tank is empty. With the additional two automata, the model can be proven to be control-loop free.

\section{NotATION AND PRELIMINARIES}

Event sequences and languages are a simple means to describe discrete system behaviours. Their basic building blocks are events, which are taken from a finite alphabet $\Sigma$. Then, $\Sigma^{+}$denotes the set of all finite strings of the form $\sigma_{1} \sigma_{2} \cdots \sigma_{k}$ of events from $\Sigma$, not including the empty string $\varepsilon$. To include it, $\Sigma^{*}=\Sigma^{+} \cup\{\varepsilon\}$ is used. The catenation of two strings $s, t \in \Sigma^{*}$ is written as st. A string $t \in \Sigma^{*}$ is called a prefix of $s \in \Sigma^{*}$, written $t \sqsubseteq s$, if $s=t u$ for some $u \in \Sigma^{*}$.

A language over $\Sigma$ is any subset $\mathcal{L} \subseteq \Sigma^{*}$. The prefixclosure $\overline{\mathcal{L}}$ of $\mathcal{L} \subseteq \Sigma^{*}$ is the set of all prefixes of strings in $\mathcal{L}$,

$$
\overline{\mathcal{L}}=\left\{t \in \Sigma^{*} \mid t \sqsubseteq s \text { for some } s \in \mathcal{L}\right\}
$$

If $\mathcal{L}=\overline{\mathcal{L}}$, then $\mathcal{L}$ is called prefix-closed.

Automata are used as a simple means of language representation. A (finite-state) automaton is a 4-tuple $A=$ $(\Sigma, Q, \Delta, J)$ where $\Sigma$ is an alphabet of events, $Q$ is a finite set of states, $\Delta \subseteq Q \times \Sigma \times Q$ is the state transition relation, and $J \subseteq Q$ is the set of initial states.

A path in $A$ is an alternating sequence of states and events

$$
\pi=q_{0} \stackrel{\sigma_{1}}{\longrightarrow} \cdots \stackrel{\sigma_{n}}{\longrightarrow} q_{n},
$$

where $\left(q_{i}, \sigma_{i+1}, q_{i+1}\right) \in \Delta$ for all $0 \leq i<n$. State $q_{0}$ is called its origin and state $q_{n}$ its end. $\mathcal{L}(\pi)=\sigma_{1} \cdots \sigma_{n}$ is the label of path $\pi$. A state $q \in Q$ is reachable in $A$ if there exists a path with origin $p \in J$ and end $q$. A path $q \stackrel{\sigma}{\rightarrow} q$ is called a selfloop, and if it exists, event $\sigma$ is said to be selflooped in $q$. To simplify the graphical representation of automata (fig. 1), events selflooped in all states of an automaton are not shown.

The language accepted by the automaton $A$ is

$$
\mathcal{L}(A)=\{\mathcal{L}(\pi) \mid \pi \text { is a path in } A \text { with origin in } J\} .
$$

Two automata over the same alphabet can be combined to a new automaton by means of the synchronous product operation [13]. The synchronous product of automata $A=\left(\Sigma, Q_{A}, \Delta_{A}, J_{A}\right)$ and $B=\left(\Sigma, Q_{B}, \Delta_{B}, J_{B}\right)$ is the automaton

$$
A \| B=\left(\Sigma, Q_{A} \times Q_{B}, \Delta, J_{A} \times J_{B}\right)
$$

where

$$
\begin{aligned}
\Delta=\{ & \left(\left(p_{1}, q_{1}\right), \sigma,\left(p_{2}, q_{2}\right)\right) \mid \\
& \left.\left(p_{1}, \sigma, p_{2}\right) \in \Delta_{A},\left(q_{1}, \sigma, q_{2}\right) \in \Delta_{B}\right\} .
\end{aligned}
$$

Note that $\mathcal{L}(A \| B)=\mathcal{L}(A) \cap \mathcal{L}(B)[1]$. 


\section{TERMINATION AND LOOPS}

The concept of control-loops relies on a set of control actions, represented by a subset $\Xi \subseteq \Sigma$ of the event alphabet. Control actions are understood to be commands caused by a controller, and it is usually desired that the sequence of such commands terminates after a finite number of steps.

This requirement is now introduced under the name of $\Xi$ termination, and it is shown that it corresponds to the absence of $\Xi$-loops in an automaton. The latter problem can be dealt with using standard graph-theoretic algorithms.

Definition 1: Let $\mathcal{L} \subseteq \Sigma^{*}$ be a prefix-closed language, and let $\Xi \subseteq \Sigma$. Then $\mathcal{L}$ is $\Xi$-terminating if for all $s \in \mathcal{L}$ there exists $n \in \mathbb{N}$ such that $s t \in \mathcal{L}$ with $|t| \geq n$ implies $t \notin \Xi^{*}$

For a $\Xi$-terminating language, there is no infinite sequence consisting of events contained in $\Xi$ only. Then, assuming that only events contained in $\Xi$ occur, the system will eventually stabilise, i.e., it will reach a state in which only events not contained in $\Xi$ are possible.

Definition 2: Let $A=(\Sigma, Q, \Delta, J)$ be an automaton, and let $\Xi \subseteq \Sigma$. A path $\pi$ in $A$ is called a $\Xi$-path if $\mathcal{L}(\pi) \in \Xi^{*}$. A $\Xi$-loop is a $\Xi$-path of length at least one with the same origin and end. A $\Xi$-loop is reachable in $A$ if its origin is reachable in $A$. If $A$ does not contain any reachable $\Xi$-loop, $A$ is called $\Xi$-loop free.

Proposition 1: Let $A=(\Sigma, Q, \Delta, J)$ be an automaton, and let $\Xi \subseteq \Sigma$. $A$ is $\Xi$-loop free if and only if $\mathcal{L}(A)$ is $\Xi$ terminating.

Proof: (Only If) Assume $\mathcal{L}(A)$ is not $\Xi$-terminating. Then there exists $s \in \mathcal{L}(A)$ such that, for each $n \in \mathbb{N}$, there exists $t \in \Xi^{*}$ such that $|t| \geq n$ and $s t \in \mathcal{L}(A)$. In particular, there exists $t \in \Xi^{*}$ such that $|t|>|Q|$ and $s t \in \mathcal{L}(A)$. Since $s t \in \mathcal{L}(A)$, there exists a path $q_{0} \stackrel{\sigma_{1}}{\longrightarrow} \cdots \stackrel{\sigma_{n}}{\longrightarrow} q_{n}$ with $q_{0} \in J$ and $\sigma_{1} \cdots \sigma_{n}=$ st. Since $|t|>|Q|$ there exists $|s| \leq i<k \leq n$ such that $q_{i}=q_{k}$. Therefore the subpath $q_{i} \stackrel{\sigma_{i+1}}{\longrightarrow} \ldots \stackrel{\sigma_{k}}{\longrightarrow} q_{k}$ is a reachable $\Xi$-loop in $A$.

(If) Assume $A$ is not $\Xi$-loop free. Let $\pi$ be a reachable $\Xi$-loop in $A$ with origin $q \in Q$. Since $\pi$ is reachable, there exists a path $\pi^{\prime}$ in $A$ with end $q$. Let $s=\mathcal{L}\left(\pi^{\prime}\right)$ and $t=$ $\mathcal{L}(\pi)$. Note that $|t| \geq 1$. Then for each $k \in \mathbb{N}$ it holds that $s t^{k} \in \mathcal{L}(A), t^{k} \in \Xi^{*}$, and $\left|t^{k}\right| \geq k$. This shows that $\mathcal{L}(A)$ is not $\Xi$-terminating.

$\Xi$-loops can be identified by finding strongly $\Xi$-connected components and selfloops labelled with events contained in $\Xi$. In order to define the concept of strongly $\Xi$-connected components, a relation over states is defined: two states are strongly $\Xi$-connected if they can mutually be reached by means of events contained in $\Xi$.

Definition 3: Let $A=(\Sigma, Q, \Delta, J)$ be an automaton, and let $\Xi \subseteq \Sigma$. Two states $p, q \in Q$ are strongly $\Xi$-connected in $A$, written $p \leftrightarrow \Xi q$, if there exist a $\Xi$-path with origin $p$ and end $q$ and another $\Xi$-path with origin $q$ and end $p$.

It can easily be shown that this relation is an equivalence relation. Its equivalence classes are called strongly $\Xi$-connected components. Such an equivalence class contains states where each state can be reached by means of $\Xi$-paths from all other states in the equivalence class.
Definition 4: Let $A=(\Sigma, Q, \Delta, J)$ be an automaton, and let $\Xi \subseteq \Sigma$. The equivalence classes of $\leftrightarrow \Xi$ are called the strongly $\Xi$-connected components of $A$. A strongly $\Xi$ connected component is said to be reachable in $A$, if it contains a state that is reachable in $A$.

The next result states that, if an automaton has a strongly $\Xi$-connected component with more than one state, then there exists a $\Xi$-loop in the automaton.

Proposition 2: Let $A=(\Sigma, Q, \Delta, J)$ be an automaton, and let $\Xi \subseteq \Sigma$. If there exist $p, q \in Q$ such that $p \leftrightarrow \Xi q$ and $p \neq q$, then there exists a $\Xi$-loop in $A$.

Proof: Since $p \leftrightarrow \Xi q$, there exist two $\Xi$-paths, one with origin $p$ and end $q$ and another with origin $q$ and end $p$. By concatenating these two paths, a $\Xi$-loop in $A$ is obtained.

If a strongly $\Xi$-connected component is reachable, then each of its states is reachable. Therefore, the loop constructed in proposition 2 is also reachable. Thus, if there exists a reachable strongly $\Xi$-connected component in an automaton, then the automaton cannot be $\Xi$-loop free.

Iterative $\Xi$-pairs are counterexamples, which can be provided when an automaton is not $\Xi$-loop free. An iterative $\Xi$-pair consists of two strings, where the second string can be iterated while staying within the behaviour of the system.

Definition 5: Let $A=(\Sigma, Q, \Delta, J)$ be an automaton, and let $\Xi \subseteq \Sigma$. The pair $(s, t) \in \Sigma^{*} \times \Xi^{+}$is an iterative $\Xi$-pair in $A$ if $s t^{n} \in \mathcal{L}(A)$ for all $n \in \mathbb{N}$.

If there exists a reachable $\Xi$-loop $\pi$ in an automaton, then there exists an iterative $\Xi$-pair in this automaton. This can be seen as follows. Since $\pi$ is reachable, there exists a path $\pi^{\prime}$ to the origin of $\pi$. Then $\left(\mathcal{L}\left(\pi^{\prime}\right), \mathcal{L}(\pi)\right)$ is an iterative $\Xi$-pair in the automaton. The first string shows how a loop can be reached. The second string describes the loop. This provides illustrative information why a system is not $\Xi$-loop free.

The results given so far suggest an algorithm to check an automaton for $\Xi$-loops. Given an automaton $A=$ $(\Sigma, Q, \Delta, J)$ and a set of control actions $\Xi \subseteq \Sigma$, there are only two possible ways how a $\Xi$-loop can exist.

(i) There is a reachable state $q \in Q$ and a control action $\xi \in \Xi$ such that $(q, \xi, q) \in \Delta$. Then $q \stackrel{\xi}{\rightarrow} q$ is a reachable $\Xi$-loop in $A$.

(ii) There exists a reachable strongly $\Xi$-connected component of $A$ consisting of at least two states $p, q \in Q$. Then there exist $\Xi$-paths from $p$ to $q$ and from $q$ to $p$, i.e., $A$ contains a reachable $\Xi$-loop.

If neither of these two conditions is satisfied, then $A$ is $\Xi$-loop free. This can be shown as follows. If $A$ contains a reachable $\Xi$-loop, it is either a selfloop, and thus condition (i) is satisfied, or the loop contains at least two different states. Then these states are in the same strongly $\Xi$-connected component and condition (ii) is satisfied. Therefore, $A$ cannot have a reachable $\Xi$-loop.

Thus, to check an automaton for $\Xi$ loops, it suffices to check for the existence of selfloops and for the existence of reachable strongly $\Xi$-connected components with more than one state. Efficient algorithms [14], [15] can be used to find strongly connected components in a directed graph in linear complexity (with respect to the number of nodes and edges). 


\section{EXPloiting Modularity}

The results of the previous section provide good algorithms to check whether a single automaton is $\Xi$-loop free. However, practical systems are composed of several automata, and then their synchronous composition needs to be computed first, which can quickly lead to intractably large automata. This section shows that synchronous composition can often be avoided by exploiting the modular structure of the system.

The most important result is that it suffices to find a $\Xi$-loop free subsystem to show that the whole system is $\Xi$-loop free. Therefore, if a $\Xi$-loop free subsystem can be found, there is no more need to compute any larger synchronous product.

Proposition 3: Let $A_{1}$ and $A_{2}$ be automata over the alphabet $\Sigma$, and let $\Xi \subseteq \Sigma$. If $A_{1}$ is $\Xi$-loop free then $A_{1} \| A_{2}$ is also $\Xi$-loop free.

Proof: Assume that $A_{1} \| A_{2}$ is not $\Xi$-loop free. Then $\mathcal{L}\left(A_{1} \| A_{2}\right)$ is not $\Xi$-terminating, that is, there exists $s \in$ $\mathcal{L}\left(A_{1} \| A_{2}\right) \subseteq \mathcal{L}\left(A_{1}\right)$ such that for all $n \in \mathbb{N}$ there exists $t_{n} \in \Xi^{*}$ such that $\left|t_{n}\right| \geq n$ and $s t_{n} \in \mathcal{L}\left(A_{1} \| A_{2}\right) \subseteq \mathcal{L}\left(A_{1}\right)$. But then $\mathcal{L}\left(A_{1}\right)$ is not $\Xi$-terminating. This proves that $A_{1}$ is not $\Xi$-loop free if $A_{1} \| A_{2}$ is not $\Xi$-loop free.

It is also possible to prove that a system is not $\Xi$-loop free without constructing its entire state space. Assume a counterexample, i.e., an iterative $\Xi$-pair has been found for a subsystem. If this counterexample is accepted by all automata constituting the whole system, then it is a counterexample for the entire system, i.e., the system is not $\Xi$-loop free.

Proposition 4: Let $A_{1}$ and $A_{2}$ be automata over the alphabet $\Sigma$, and let $\Xi \subseteq \Sigma$. Every iterative $\Xi$-pair in both $A_{1}$ and $A_{2}$ also is an iterative $\Xi$-pair in $A_{1} \| A_{2}$.

Proof: Let $A=A_{1} \| A_{2}$, and let $(s, t)$ be an iterative $\Xi$-pair in $A_{1}$ and $A_{2}$. Then $t \in \Xi^{+}, s t^{n} \in \mathcal{L}\left(A_{1}\right)$, and $s t^{n} \in \mathcal{L}\left(A_{2}\right)$ for each $n \in \mathbb{N}$. This implies $s t^{n} \in \mathcal{L}\left(A_{1}\right) \cap$ $\mathcal{L}\left(A_{2}\right)=\mathcal{L}(A)$. Thus, $(s, t)$ is an iterative $\Xi$-pair in $A$.

Propositions 3 and 4 can be combined to a strategy to construct subsystems iteratively. Assume a system

$$
A=A_{1}\|\cdots\| A_{n}
$$

is to be checked for $\Xi$-loops, and some subsystem $A_{i}$ of $A$ has already been identified. If $A_{i}$ is $\Xi$-loop free, then the entire system $A$ is known to be $\Xi$-loop free by proposition 3 . If $A_{i}$ is not $\Xi$-loop free, there exists an iterative $\Xi$-pair demonstrating that $A_{i}$ is not $\Xi$-loop free. If this also is an iterative $\Xi$-pair in all other components $A_{1}, \ldots, A_{n}$ of $A$, then the entire system $A$ is not $\Xi$-loop free by proposition 4 . Otherwise, there is an automaton $A_{j}$ that does not accept the iterative $\Xi$-pair, and this automaton is a good candidate to augment the subsystem $A_{i}$ and consider $A_{i} \| A_{j}$ in a next step.

Example 1: Consider the automata in fig. 1, with the set of control events

$$
\Xi=\{\text { open_in, close_in, open_out, close_out }\} \text {. }
$$

The subsystem req_spec is not $\Xi$-loop free as shown by the iterative $\Xi$-pair (req_start,open_in). This is not an iterative $\Xi$-pair in automaton no flow, so proposition 4 cannot

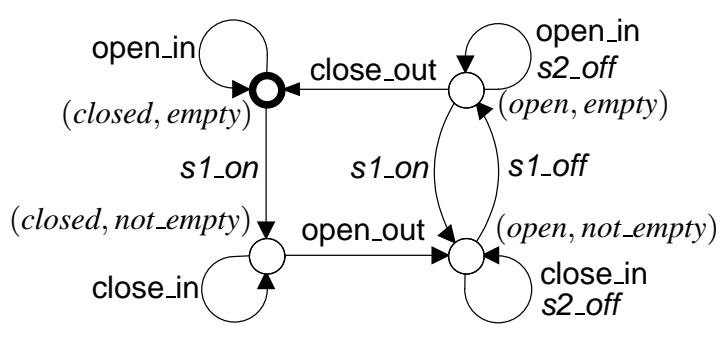

Fig. 3. The synchronous composition out $\|$ empty_spec'.

yet be applied. Therefore, the larger subsystem req_spec $\|$ no flow is considered. This yields the new iterative $\Xi$-pair (req_start, open_in close_in), which is accepted by all other automata and therefore is a counterexample for the model.

This method can be improved further. The set $\Xi$ of control events can be reduced by removing events that are known not to be contained in any $\Xi$-loop of the system. Consider an event $\xi \in \Xi$ and a subsystem such that $\xi$ is not contained in any $\Xi$-loop of this subsystem. Then the event $\xi$ cannot occur in a $\Xi$-loop of the entire system since each $\Xi$-loop in the synchronous product must also be a $\Xi$-loop in each of the automata constituting the synchronous product. Therefore, it suffices to check whether the system is $(\Xi \backslash\{\xi\})$-loop free, which is simpler than checking whether it is $\Xi$-loop free.

Proposition 5: Let $A_{1}$ and $A_{2}$ be automata over the alphabet $\Sigma$, and let $\Xi \subseteq \Sigma$. Furthermore, let $\xi \in \Xi$ such that $\xi \notin \mathcal{L}(\pi)$ for each reachable $\Xi$-loop $\pi$ of $A_{1}$. Then $A_{1} \| A_{2}$ is $\Xi$-loop free if and only if $A_{1} \| A_{2}$ is $(\Xi \backslash\{\xi\})$-loop free.

Proof: Let $A=A_{1} \| A_{2}$ and $\Xi^{\prime}=\Xi \backslash\{\xi\}$.

(Only If) Assume that $A$ is not $\Xi^{\prime}$-loop free. Then there exists a reachable $\Xi^{\prime}$-loop in $A$. This is also a reachable $\Xi$-loop in $A$, i.e., $A$ is not $\Xi$-loop free.

(If) Assume that $A$ is not $\Xi$-loop free, i.e., there exists a reachable $\Xi$-loop

$$
\pi=\left(p_{0}, q_{0}\right) \stackrel{\xi_{1}}{\longrightarrow} \cdots \stackrel{\xi_{n}}{\longrightarrow}\left(p_{n}, q_{n}\right)
$$

in $A=A_{1} \| A_{2}$. Then

$$
\pi_{1}=p_{0} \stackrel{\xi_{1}}{\longrightarrow} \cdots \stackrel{\xi_{n}}{\longrightarrow} p_{n}
$$

is a reachable $\Xi$-loop in $A_{1}$. By assumption, $\xi \notin \mathcal{L}\left(\pi_{1}\right)=$ $\mathcal{L}(\pi)$, i.e., $\mathcal{L}(\pi) \in\left(\Xi^{\prime}\right)^{*}$. Hence, $A$ is not $\Xi^{\prime}$-loop free.

Example 2: Consider the extended model of the introductory example given by the automata in fig. 1 and fig. 2 . The system can be shown to be $\Xi$-loop free as follows. Consider the synchronous product of automata out and empty_spec', shown in fig. 3. This automaton contains $\Xi$-loops, but none of them contains the events open_out or close_out. By proposition 5 , these two events can be removed from $\Xi$, i.e., it is sufficient to check for $\{$ open_in, close_in\}-loops. The same argument can be applied to the remaining two events when considering the synchronous product of automata in and fill spec'. Thereby $\Xi$ is reduced to the empty set, already proving that the entire system is control-loop free. 


\section{A Modular Algorithm}

The results from the previous section can be combined in different ways to obtain algorithms to find control loops in modular systems. The method proposed in this paper uses ideas from [8]. Given a modular system

$$
A=A_{1}\|\cdots\| A_{n},
$$

to be checked, it tries to construct a $\Xi$-loop free subsystem of $A$ incrementally, including more automata as needed.

But first, all automata $A_{i}$ are checked for $\Xi$-loops individually. Typically, none of them is $\Xi$-loop free by themselves because of implicit selfloops. Yet, by proposition 5, this first pass can identify events that cannot occur in any $\Xi$-loop and thereby considerably reduce the set $\Xi$ of control actions.

Then the construction of subsystems of $A$ begins. Starting from the empty subsystem, which accepts $\Sigma^{*}$ and therefore is never loop-free, new automata are added until a result is obtained. Assume a subsystem

$$
A^{\prime}=A_{i_{1}}\|\cdots\| A_{i_{m}}
$$

has been constructed. If $A^{\prime}$ is $\Xi$-loop free, then the entire system $A$ is $\Xi$-loop free by proposition 3. Otherwise an iterative $\Xi$-pair can be constructed. If this counterexample is an iterative $\Xi$-pair for all automata in $A$, then the entire system $A$ has a $\Xi$-loop by proposition 4 . Otherwise, some automaton does not accept the counterexample. The algorithm chooses one of the automata $A_{j}$ not accepting the counterexample, and analyses the larger subsystem

$$
A^{\prime \prime}=A^{\prime} \| A_{j}
$$

in the next step. In addition, the set of control events occurring in all $\Xi$-loops of $A^{\prime}$ is identified to apply proposition 5 and replace $\Xi$ by a smaller set when analysing $A^{\prime \prime}$.

Given a counterexample, the above algorithm looks for automata not accepting the particular counterexample to include one or more of these automata in the next analysis attempt. Performance may depend on which automaton is chosen. A number of heuristics for selecting automata not accepting a counterexample of interest have been proposed in [8] and are listed below. They have all been implemented and tested on the industrial examples of section VII.

- All. Use all automata not accepting the counterexample.

- EarlyNotAccept. Use the automaton rejecting the counterexample as early as possible, i.e., the automaton accepting as little as possible of the counterexample.

- LateNotAccept. Use the automaton rejecting the counterexample as late as possible.

- MaxCommonEvents. Use the automaton with the most events in common with the system considered so far. An automaton sharing many events with the system considered is likely to interact more closely with it, and is therefore more likely to contribute to the analysis.

- MaxCommonUncontr. Use the automaton with the most non-control events in common with the system considered so far. This is similar to MaxCommonEvents, but more tuned to the control-loop check.
- MinEvents. Use the automaton with the fewest events, in an attempt to use the simplest automata and construct the smallest possible synchronous product.

- MinNewEvents. Use the automaton adding the fewest events to the system considered. This is similar to MaxCommonEvents, which looks for an automaton interacting closely with the system considered.

- MinStates. Use the automaton with the fewest states. Similar to the MinEvents heuristic, this is an attempt to construct a small synchronous product.

- MinTransitions. Use the automaton with the fewest transitions.

- One. Use the first automaton found not to accept the counterexample.

- RelMaxCommonEvents. Use the automaton with the maximum ratio of shared events with the system considered to the number of used events. This is similar to MaxCommonEvents, but tries to avoid adding complex automata using many events, but sharing few events with the system considered.

\section{EXPERIMENTAL RESUlTS}

The proposed algorithm has been tested with a set of industrial examples and case studies previously used in [8]. All examples considered are listed below, together with the corresponding automata models, also referred to in table I.

- Case study production cell I [16]: fzelle.

- Case study production cell II [17]: ftechnik.

- PROFIsafe field bus protocol [18]-[20]: profisafe_i4, profisafe_04.

- AIP automated manufacturing system [21]-[23]: rhonealps, rhone_tough.

- Train testbed [24]: tbed_uncont, tbed_ctct, tbed_valid.

- Central locking system (KORSYS project): verriegel4.

These models have been checked for control-loops, using the proposed algorithm and each of the heuristics mentioned in section VI. All tests were performed on a standard PC with a $1.4 \mathrm{GHz}$ processor and $256 \mathrm{MB}$ of RAM. The results are shown in table I.

The first two columns list the model name and the number of automata for each model. The subsequent column pairs list for each heuristic and all examples the maximum number of automata composed and the total number of states constructed. The examples above the horizontal line in the table body are control-loop free, whereas the examples below do not satisfy this property.

In spite of the complexity of the models, they all could be shown to be control-loop free or not, requiring not more than a few seconds of CPU time for each run.

All control-loop free examples could be verified easily, never considering more than a single automaton at a time. This becomes possible by the initial step of the algorithm, which identifies the loop events of each automaton individually. Using proposition 5 , it is always possible to reduce the set of possible loop events to the empty set, completely avoiding synchronous composition. The examples that contain control-loops are only slightly harder to 
TABLE I

EXPERIMENTAL DATA FROM INDUSTRIAL EXAMPLES

\begin{tabular}{|c|c|c|c|c|c|c|c|c|c|c|c|c|c|c|c|c|c|c|c|c|c|c|c|}
\hline \multicolumn{2}{|l|}{ Model } & \multicolumn{2}{|r|}{ All } & \multicolumn{2}{|c|}{\begin{tabular}{|c|} 
Early \\
NotAccept
\end{tabular}} & \multicolumn{2}{|c|}{\begin{tabular}{|c|} 
Late \\
NotAccept
\end{tabular}} & \multicolumn{2}{|c|}{$\begin{array}{c}\text { MaxCommon } \\
\text { Events }\end{array}$} & \multicolumn{2}{|c|}{$\begin{array}{c}\text { MaxCommon } \\
\text { Uncontr }\end{array}$} & \multicolumn{2}{|c|}{$\begin{array}{c}\text { Min } \\
\text { Events }\end{array}$} & \multicolumn{2}{|c|}{\begin{tabular}{|c|} 
Min \\
NewEvents
\end{tabular}} & \multicolumn{2}{|c|}{$\begin{array}{c}\text { Min } \\
\text { States }\end{array}$} & \multicolumn{2}{|c|}{$\begin{array}{c}\text { Min } \\
\text { Transitions }\end{array}$} & \multicolumn{2}{|c|}{ One } & \multicolumn{2}{|c|}{\begin{tabular}{|c|} 
RelMax \\
Common
\end{tabular}} \\
\hline Name & Aut & Aut & States & Aut & States & Aut & States & Aut & States & Aut & States & Aut & States & Aut & States & Aut & States & Aut & States & Aut & States & Aut & States \\
\hline profi safe i4 & 75 & 1 & 267 & 1 & 267 & 1 & 267 & 1 & 267 & 1 & 267 & & 267 & 1 & 267 & 1 & 267 & 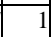 & 267 & & 267 & 1 & 267 \\
\hline profi safe 04 & 84 & 1 & 292 & 1 & 292 & 1 & 292 & 1 & 292 & 1 & 292 & 1 & 292 & . & 292 & 1 & 292 & 1 & 292 & 1 & 292 & 1 & 292 \\
\hline rhone_tough & 61 & 1 & 318 & 1 & 318 & 1 & 318 & 1 & 318 & 1 & 318 & 1 & 318 & 1 & 318 & 1 & 318 & 1 & 318 & 1 & 318 & 1 & 318 \\
\hline tbed_ctct & 84 & 1 & 507 & 1 & 507 & 1 & 507 & 1 & 507 & 1 & 507 & 1 & 507 & 1 & 507 & 1 & 507 & 1 & 507 & 1 & 507 & 1 & 507 \\
\hline tbed_uncont & 58 & 1 & 421 & 1 & 421 & 1 & 421 & 1 & 421 & 1 & 421 & 1 & 421 & 1 & 421 & 1 & 421 & 1 & 421 & 1 & 421 & 1 & 421 \\
\hline tbed_valid & 84 & 1 & 468 & 1 & 468 & 1 & 468 & 1 & 468 & 1 & 468 & 1 & 468 & 1 & 468 & 1 & 468 & 1 & 468 & 1 & 468 & 1 & 468 \\
\hline verriegel4 & 65 & 1 & 726 & 1 & 726 & 1 & 726 & 1 & 726 & 1 & 726 & 1 & 726 & 1 & 726 & 1 & 726 & 1 & 726 & 1 & 726 & 1 & 726 \\
\hline ftechnik & 36 & 4 & 2927 & 4 & 3053 & 4 & 3053 & 4 & 3136 & 4 & 3136 & 4 & 3053 & 4 & 3053 & 4 & 3053 & 4 & 3053 & 4 & 3053 & 4 & 3136 \\
\hline fzelle & 67 & 3 & 830 & 2 & 834 & 2 & 834 & 2 & 834 & 2 & 834 & 2 & 834 & 2 & 834 & 2 & 834 & 2 & 834 & 2 & 834 & 2 & 830 \\
\hline rhone_alps & 35 & 2 & 247 & 2 & 247 & 2 & 247 & 2 & 247 & 2 & 247 & 2 & 247 & 2 & 247 & 2 & 247 & 2 & 247 & 2 & 247 & 2 & 247 \\
\hline
\end{tabular}

check: their faults are not so conspicuous that they can be found considering only one automaton. All results are largely independent of the heuristics for the selection of automata based on counterexamples.

These examples suggest that models of practical systems are typically well-structured in a way that makes modular verification easy. Control-loops can be detected automatically very quickly, making this property an excellent candidate to be implemented as a push-button feature in development tools that support engineers in the design of reactive systems.

\section{CONCLUSIONS}

An efficient algorithm to check a finite-state system for control-loops has been presented. This algorithm can avoid explicit state exploration of the entire system state space by exploiting the modularity common in many practical applications. Experimental results show that the new method can quickly verify several examples of industrial scale.

The proposed algorithm can be extended and enhanced in many ways. In the future, the authors would like to experiment with different heuristics for the selection of automata, and to investigate more elaborate reasoning. With the possibility to reduce the set of possible loop events, it is not required to keep increasing the subsystem being checked. It may be more efficient to restart with smaller systems when the set of loop events has been reduced.

\section{REFERENCES}

[1] P. J. G. Ramadge and W. M. Wonham, "The control of discrete event systems," Proc. IEEE, vol. 77, no. 1, pp. 81-98, Jan. 1989.

[2] C. G. Cassandras and S. Lafortune, Introduction to Discrete Event Systems. Kluwer, Sept. 1999.

[3] S. Balemi, G. J. Hoffmann, P. Gyugyi, H. Wong-Toi, and G. F. Franklin, "Supervisory control of a rapid thermal multiprocessor," IEEE Trans. Automat. Contr., vol. 38, no. 7, pp. 1040-1059, July 1993.

[4] S. Balemi, "Input/output discrete event processes and communication delays," Discrete Event Dynamic Systems: Theory and Applications, vol. 4, pp. 41-85, 1994.

[5] C. H. Golaszewski and P. J. Ramadge, "Control of discrete event processes with forced events," in Proc. 26th IEEE Conf. Decision and Control, CDC'87, Los Angeles, CA, USA, Dec. 1987, pp. 247-251.

[6] P. Dietrich, R. Malik, W. M. Wonham, and B. A. Brandin, "Implementation considerations in supervisory control," in Synthesis and Control of Discrete Event Systems, B. Caillaud, P. Darondeau, L. Lavagno, and X. Xie, Eds. Kluwer, 2002, pp. 185-201.
[7] P. Malik, "From supervisory control to nonblocking controllers for discrete event systems," Ph.D. dissertation, University of Kaiserslautern, Kaiserslautern, Germany, 2003.

[8] B. A. Brandin, R. Malik, and P. Malik, "Incremental verifi cation and synthesis of discrete-event systems guided by counter-examples," IEEE Trans. Contr. Syst. Technol., vol. 12, no. 3, pp. 387-401, May 2004.

[9] E. M. Clarke, E. A. Emerson, and A. P. Sistla, "Automatic verifi cation of fi nite-state concurrent systems using temporal logic specifi cations," ACM Trans. Programming Languages and Systems, vol. 8, no. 2, pp. 244-263, Apr. 1986

[10] K. L. McMillan, Symbolic Model Checking. Kluwer, 1993.

[11] E. M. Clarke, Jr., O. Grumberg, and D. A. Peled, Model Checking. MIT Press, 1999.

[12] H.-M. Hanisch and S. Kowalewski, "Algebraic synthesis and verifi cation of discrete supervisory controllers for forbidden path specifi cations," in Proc. 4th Int. Conf. Computer Integrated Manufacturing and Automation Technology. Troy, NY, USA: IEEE Computer Society Press, Oct. 1994, pp. 157-162.

[13] C. A. R. Hoare, Communicating Sequential Processes. Prentice-Hall, 1985.

[14] R. Tarjan, "Depth fi rst search and linear graph algorithms," SIAM J. Computing, vol. 1, no. 2, pp. 146-160, June 1972.

[15] E. Nuutila and E. Soisalon-Soininen, "On fi nding the strongly connected components in a directed graph," Information Processing Letters, vol. 49, no. 1, pp. 9-14, Jan. 1994.

[16] C. Lewerentz and T. Linder, Case Study "Production Cell", ser. LNCS. Springer, 1995, vol. 891.

[17] A. Lötzbeyer and R. Mühlfeld, "Task description of a flexible production cell with real time properties," FZI, Karlsruhe, Germany, Tech. Rep., 1996. [Online]. Available: http://www.fzi.de/divisions/prost/projects/korsys/korsys.html

[18] R. Malik and R. Mühlfeld, "A case study in verifi cation of UML statecharts: the PROFIsafe protocol," J. Universal Computer Science, vol. 9, no. 2, pp. 138-151, Feb. 2003.

[19] - " "Testing the PROFIsafe protocol using automatically generated test cases based on a formally verifi ed model," Siemens AG, Corporate Technology, Software and Engineering 1, Munich, Germany, Tech. Rep., 2002.

[20] Profi bus Nutzerorganisation e. V., "PROFIsafe—profi le for safety technology, version 1.12," 2002.

[21] B. Brandin and F. Charbonnier, "The supervisory control of the automated manufacturing system of the AIP," in Proc. Rensselaer's 4th Int. Conf. Computer Integrated Manufacturing and Automation Technology, Troy, NY, USA, 1994, pp. 319-324.

[22] F. Charbonnier, "Commande par supervision des systèmes à événements discrets: application à un site expérimental l'Atelier Interétablissement de Productique," Laboratoire d'Automatique de Grenoble, Grenoble, France, Tech. Rep., 1994.

[23] R. J. Leduc, "Hierarchical interface-based supervisory control," Ph.D. dissertation, Dept. of Electrical Engineering, University of Toronto, Ontario, Canada, 2002.

[24] — - "PLC implementation of a DES supervisor for a manufacturing testbed: An implementation perspective," Master's thesis, Dept. of Electrical Engineering, University of Toronto, Ontario, Canada, 1996. 